\title{
PRODUCTION POTENTIAL AND ECONOMICS OF HYBIRD RICE UNDER SYSTEM OF RICE INTENSIFICATION AND ITS MANIPULATION
}

\author{
A. K. Verma*, N. Pandey and G. K. Shrivastava \\ Department of Agronomy, Indira Gandhi Krishi Vishwavidyalaya, Chhattisgarh, India
}

\begin{abstract}
The study was conducted at Indira Gandhi Krishi Vishwavidyalaya, Chhattisgarh $\left(21^{\circ} 16^{\prime} \mathrm{N}\right.$ and $\left.81^{\circ} 36^{\prime} \mathrm{E}\right)$ during wet season of 2007-08 and 2008-09 in silty clay loam soil to assess the effects of system of rice intensification (SRI) and its manipulation involving two age of seedlings (10 and 14 days), three manuring (organic alone, inorganic alone, 50\% nutrients through organic $+50 \%$ nutrients through inorganic), two weeding practices (Mechanical weeding through conoweeder and chemical weeding), two water management practices (application of 2 $\mathrm{cm}$ water at hairline crack development stage and cyclic submergence of $5 \mathrm{~cm}$ water at 3 days after disappearance of ponded water) and one local recommended practices on hybrid rice (Oryza sativa L.). The results revealed manipulated SRI (10 days aged seedlings $+100 \%$ nutrients through inorganic or $50 \%$ through organic $+50 \%$ through inorganic + irrigation as per SRI) gave $13.52 \%$ higher grain yield and $16.80 \%$ higher net income over recommended practices of hybrid rice.
\end{abstract}

Key words: INM, Inorganic, Mechanical weeding, Organic, Seedling age, SRI

\section{INTRODUCTION}

Rice plays a pivotal role in ensuring food security of India. Rice alone contributes to $43 \%$ of total food grain production and $46 \%$ of the total cereal production. India is largest rice growing county in the world but productivity is very low $\left(1.94 \mathrm{t} \mathrm{ha}^{-1}\right)$ as compared to China $\left(4.24 \mathrm{t} \mathrm{ha}^{-1}\right)$. Appropriate set of agronomical management is required for increasing the productivity. SRI i.e. system of rice intensification has been proved to be one of the sound and promising agronomic practice in enhancing the productivity of rice (Uphoff, 2001). Hybrid rice is although gaining popularity among the Indian farmers but its potential is yet to be exploited fully through hybrid rice cultivation. The hybrid rice offers $10-15 \%$ yield advantage

\footnotetext{
*Corresponding author email: dranilverma1973@gmail.com
} 
over the best conventional inbred varieties which may further enhanced by adoption of SRI due to use of early seedling and maintaining uniform geometry.

System of rice intensification (SRI) originated in Madagascar and by adopting this method average rice yield can be about the double without changing the cultivars or the use of purchased input (Wang et al., 2003). SRI is based on the assumptions that micro scale modification of soil, water and nutrient management practices which may be suppressed by crop when growing as irrigated rice. SRI methods appears to be promising, but it is essential to test whether the agronomic practices recommended holds good for our agro ecological conditions as well. Its quantitative analysis will have to carry out and factors responsible for higher yield are to be identified. This information helps for adoptability and increasing the regional productivity, which is presently lower than the national average. Moreover, adoption of all the principles of SRI are also not a easy task, therefore, it is essential to alter these in view of existing agro climatic condition and resource availability of the farmers. Keeping in view, the present investigation was conducted to assess the production potential and economics of hybrid rice under system of rice intensification and its manipulation.

\section{MATERIALS AND METHODS}

Field experiment was carried out during kharif 2007-08 and 2008-09 at Research cum Instructional farm of Indira Gandhi Krishi Vishwavidyalaya, Raipur, Chhattisgarh. The soil of the experimental field was deep clay loam in texture and taxonomically classified as typic Haplustaff. The soil was low in available N $(214 \mathrm{~kg}$ $\left.\mathrm{N} \mathrm{ha}^{-1}\right)$, medium in available $\mathrm{P}\left(18.8 \mathrm{~kg} \mathrm{P} \mathrm{ha}^{-1}\right)$ and high in available $\mathrm{K}(293 \mathrm{~kg} \mathrm{~K}$ ha $\left.{ }^{1}\right)$. The electrical conductivity of the soil was $0.51 \mathrm{ds} \mathrm{m}^{-1}$ and the $\mathrm{pH}$ was 7.3 . Mechanical analysis of the soil showed $6.92,13.55,43.22$ and $35.81 \%$ of coarse sand, fine sand, silt and clay, respectively. The rice hybrid Proagro 6444 was used in trial as test crop with field duration of 120 days.

Poultry manure and FYM were used as a source of organic manure. The poultry manure and FYM were applied to the puddle soil and was incorporated manually. The NPK content in FYM and poultry manure on dry weight basis were $1.41,0.51,1.80$ and 2.66, 1.50, 2.01, respectively. In all $7 \mathrm{t} \mathrm{ha}^{-1}$ of FYM and $2.5 \mathrm{tha}^{-1}$ poultry manure was applied before the transplanting as per the treatments. Fertilizers were applied at the rate of $150-75-60 \mathrm{~kg} \mathrm{ha}^{-1}$ of $\mathrm{N}, \mathrm{P}_{2} \mathrm{O}_{5}, \mathrm{~K}_{2} \mathrm{O} \mathrm{ha}^{-1}$ (convert PK), respectively in the form of urea, single super phosphate and muriate of potash. The whole amount of phosphorus and potassium was applied as basal, whereas, nitrogen was applied as 40 per cent as basal, 25 per cent at tillering, 25 per cent at panicle initiation and 10 per cent at flowering stages. The experiment was laid out in a randomized block design with three replications. Data were collected on number of weeds / m2, flag leaf number of leaves and dry matter at, 54, 65, 54 and 90 DAT. Economic analysis was also done on the basis of prevailing market price. The details of the treatments and the notations used are as follows: 
Table 1: Treatment details of the experiment

\begin{tabular}{|c|c|c|}
\hline Treatment & Treatment details & $\begin{array}{l}\text { Notations } \\
\text { used }\end{array}$ \\
\hline $\mathrm{T}_{1}$ & $\begin{array}{l}10 \text { days seedlings }+100 \% \text { manure }+ \text { Mechanical weed } \\
\text { management }+ \text { Application of } 2 \mathrm{~cm} \text { water at hairline crack } \\
\text { development stage (SRI) }\end{array}$ & $\mathrm{A}_{10} \mathrm{M}_{100} \mathrm{~W}_{\mathrm{m}} \mathrm{I}_{\mathrm{s}}$ \\
\hline $\mathrm{T}_{2}$ & $\begin{array}{l}10 \text { days seedlings }+100 \% \text { manure }+ \text { Chemical weed } \\
\text { management + Irrigation as per SRI }\end{array}$ & $\mathrm{A}_{10} \mathrm{M}_{100} \mathrm{~W}_{\mathrm{c}} \mathrm{I}_{\mathrm{s}}$ \\
\hline $\mathrm{T}_{3}$ & $\begin{array}{l}10 \text { days seedlings }+100 \% \text { manure }+ \text { Mechanical weed } \\
\text { management }+ \text { Cyclic submergence of } 5 \mathrm{~cm} \text { water at } 3 \text { days } \\
\text { after disappearance }(\mathrm{DAD}) \text { of ponded water }\end{array}$ & $\mathrm{A}_{10} \mathrm{M}_{100} \mathrm{~W}_{\mathrm{m}} \mathrm{I}_{3 \mathrm{D}}$ \\
\hline $\mathrm{T}_{4}$ & $\begin{array}{l}14 \text { days seedlings }+100 \% \text { manure }+ \text { Mechanical weed } \\
\text { management + Irrigation as per SRI }\end{array}$ & $\mathrm{A}_{14} \mathrm{M}_{100} \mathrm{~W}_{\mathrm{m}} \mathrm{I}_{\mathrm{s}}$ \\
\hline $\mathrm{T}_{5}$ & $\begin{array}{l}14 \text { days seedlings }+100 \% \text { manure }+ \text { Mechanical weed } \\
\text { management }+ \text { Cyclic submergence at } 3 \text { DAD of ponded } \\
\text { water }\end{array}$ & $\mathrm{A}_{14} \mathrm{M}_{100} \mathrm{~W}_{\mathrm{m}} \mathrm{I}_{3 \mathrm{D}}$ \\
\hline $\mathrm{T}_{6}$ & $\begin{array}{l}10 \text { days seedlings }+100 \% \text { fertilizer }+ \text { Mechanical weed } \\
\text { management }+ \text { Irrigation as per SRI }\end{array}$ & $\mathrm{A}_{10} \mathrm{~F}_{100} \mathrm{~W}_{\mathrm{m}} \mathrm{I}_{\mathrm{s}}$ \\
\hline $\mathrm{T}_{7}$ & $\begin{array}{l}10 \text { days seedlings }+100 \% \text { fertilizer }+ \text { Chemical weed } \\
\text { management + Irrigation as per SRI }\end{array}$ & $\mathrm{A}_{10} \mathrm{~F}_{100} \mathrm{~W}_{\mathrm{c}} \mathrm{I}_{\mathrm{s}}$ \\
\hline $\mathrm{T}_{8}$ & $\begin{array}{l}14 \text { days seedlings }+100 \% \text { fertilizer }+ \text { Mechanical weed } \\
\text { management }+ \text { Irrigation as per SRI }\end{array}$ & $\mathrm{A}_{14} \mathrm{~F}_{100} \mathrm{~W}_{\mathrm{m}} \mathrm{I}_{\mathrm{s}}$ \\
\hline $\mathrm{T}_{9}$ & $\begin{array}{l}10 \text { days seedlings }+50 \% \text { manure }+50 \% \text { fertilizer }+ \\
\text { Mechanical weed management }+ \text { Irrigation as per SRI }\end{array}$ & $\mathrm{A}_{10} \mathrm{M}_{50} \mathrm{~F}_{50} \mathrm{~W}_{\mathrm{m}} \mathrm{I}$ \\
\hline $\mathrm{T}_{10}$ & $\begin{array}{l}10 \text { days seedlings }+50 \% \text { manure }+50 \% \text { fertilizer }+ \\
\text { Chemical weed management }+ \text { Irrigation as per SRI }\end{array}$ & $\mathrm{A}_{10} \mathrm{M}_{50} \mathrm{~F}_{50} \mathrm{~W}_{\mathrm{c}} \mathrm{I}_{\mathrm{s}}$ \\
\hline $\mathrm{T}_{11}$ & $\begin{array}{l}14 \text { days seedlings }+50 \% \text { manure }+50 \% \text { fertilizer }+ \\
\text { Mechanical weed management + Irrigation as per SRI }\end{array}$ & $\mathrm{A}_{14} \mathrm{M}_{50} \mathrm{~F}_{50} \mathrm{~W}_{\mathrm{m}} \mathrm{I}$ \\
\hline $\mathrm{T}_{12}$ & $\begin{array}{l}\text { Recommended practices of hybrid rice ( } 21 \text { days seedling }+ \\
100 \% \text { fertilizer }+ \text { Two hand weeding at } 20 \text { and } 40 \text { DAT }+ \\
\text { Cyclic submergence at } 3 \text { DAD of ponded water) }\end{array}$ & $\mathrm{A}_{21} \mathrm{~F}_{100} \mathrm{~W}_{\mathrm{H}} \mathrm{I}_{3 \mathrm{D}}$ \\
\hline
\end{tabular}

A-Age of seedling; M-Manure; F-Fertilizer; $\mathrm{W}_{\mathrm{m}}, \mathrm{W}_{\mathrm{c}}$ \& $\mathrm{W}_{\mathrm{H}}-$ Mechanical, chemical \& hand weeding, respectively; $\mathrm{I}_{\mathrm{s}} \& \mathrm{I}_{3 \mathrm{D}^{-}}$- Irrigation as per SRI \& at $3 \mathrm{DAD}$, respectively 
A. K. Verma et al.

\section{RESULTS AND DISCUSSION}

\section{Growth characters}

Treatments involved in manipulation of different crop management and inputs i.e. seedling age, nutrient, water and weed management practices of SRI revealed that the planting of 10 days aged seedlings $+100 \%$ nutrients applied through inorganic + mechanical weeding + irrigation as per SRI $\left(\mathrm{A}_{10} \mathrm{~F}_{100} \mathrm{~W}_{\mathrm{m}} \mathrm{I}_{\mathrm{s}}\right)$ produced the tallest plant $(120.10 \mathrm{~cm})$ and maintained its superiority over other treatments except planting of 10 days aged seedlings $+50 \%$ nutrients applied through inorganic $+50 \%$ nutrients applied through organic + mechanical weeding + irrigation as per SRI $\left(\mathrm{A}_{10} \mathrm{M}_{50} \mathrm{~F}_{50} \mathrm{~W}_{\mathrm{m}} \mathrm{I}_{\mathrm{s}}\right)$ (Table 1). Transplanting of younger seedlings (10 days aged) supported with sufficient nutrients and aerated soil condition favoured the plant growth and increased plant height, number of leaves, flag leaf area, SPAD value, number and weight of tillers, root and shoot dry biomass because of higher cell division and cell enlargement resultant of more photosynthetic rate as also reported by Krishna (2000), Shrirame et al. (2000) and Lokanadhan et al. (2007).

\section{Weed density and biomass}

Weed density and biomass was highest under $\mathrm{A}_{10} \mathrm{M}_{100} \mathrm{~W}_{\mathrm{c}} \mathrm{I}_{\mathrm{S}}$ at harvest due to reduction of toxic effect of herbicide with the advancement of crop age resulting in increase in weed growth (Table 1). Similar results also reported by Mitra et al. (2005). Whereas, under mechanical weeding through Cono weeder weeds were incorporated from both the directions of the crop resulted in reduction of weed density and biomass $\left(\mathrm{A}_{10} \mathrm{~F}_{100} \mathrm{~W}_{\mathrm{m}} \mathrm{I}_{\mathrm{S}}\right.$ or $\mathrm{A}_{10} \mathrm{M}_{50} \mathrm{~F}_{50} \mathrm{~W}_{\mathrm{m}} \mathrm{I}_{\mathrm{S}}$ or $\left.\mathrm{A}_{10} \mathrm{M}_{100} \mathrm{~W}_{\mathrm{m}} \mathrm{I}_{\mathrm{S}}\right)$. Moreover, number and weight of tillers also increased under these treatments suggests that more area occupied by rice plant suppressed the weed density and growth. This is in accordance with the findings of Vijaykumar et al. (2006).

\section{Yield attributes and yield}

The combination of 10 days aged seedlings, $100 \%$ nutrients through inorganic, incorporation of weeds by mechanical weeder and irrigation as per SRI $\left(\mathrm{A}_{10} \mathrm{~F}_{100} \mathrm{~W}_{\mathrm{m}} \mathrm{I}_{\mathrm{S}}\right)$ produced the maximumgrain yield, which was at par to $\mathrm{A}_{10} \mathrm{M}_{50} \mathrm{~F}_{50} \mathrm{~W}_{\mathrm{m}} \mathrm{I}_{\mathrm{S}}$ (Table 2). The combination of $\mathrm{A}_{10} \mathrm{~F}_{100} \mathrm{~W}_{\mathrm{m}} \mathrm{I}_{\mathrm{s}}$ produced higher grain yield $\left(7.52 \mathrm{t} \mathrm{ha}^{-1}\right)$ than recommended practices of hybrid rice $\left(6.50 \mathrm{t} \mathrm{ha}^{-1}\right)$ with the increase in grain yield was $13.52 \%$. These results are in agreement with the findings of Krishna et al. (2008). Almost all yields attributes (effective tillers, weight of panicle and fertile grains panicle ${ }^{-1}$ ) except weight of 1000- grain and sterility percentage were favourably influenced by these treatments. This might be due to efficient utilization of resources and less inter and intra space competition among plants which may be assigned as the reason for superiority in these yield attributes of hybrid rice and consequently increased yield. Padmavati et al. (1998) also reported the similar findings. The lower yield was recorded under the treatment of $\mathrm{A}_{10} \mathrm{M}_{100} \mathrm{~W}_{\mathrm{c}} \mathrm{I}_{\mathrm{s}}$. A decrease in leaf area causes a reduction in area for interception and 
absorption of the specific wavelength of light necessary for photosynthesis resulted in reduction of root and plant dry biomass thereby reducing absorption of nutrients subsequently reducing the yield attributes and yield.

\section{Water requirement}

The total water requirement $(823 \mathrm{~mm})$ was increased where irrigation applied as per SRI i.e., $2 \mathrm{~cm}$ water at hairline crack development stage $\left(\mathrm{A}_{10} \mathrm{~F}_{100} \mathrm{~W}_{\mathrm{m}} \mathrm{I}_{\mathrm{S}}\right)$ due to more frequency of irrigation than irrigation applied at $3 \mathrm{DAD}$ i.e. cyclic submergence of $5 \mathrm{~cm}$ water at 3 days after disappearance of ponded water $\left(\mathrm{A}_{21} \mathrm{~F}_{100} \mathrm{~W}_{\mathrm{H}} \mathrm{I}_{3 \mathrm{D}}\right)$ (Table $1)$.

\section{Economics}

The maximum net income was received under $\mathrm{A}_{10} \mathrm{~F}_{100} \mathrm{~W}_{\mathrm{m}} \mathrm{I}_{\mathrm{S}}$ which was at par with the treatment of $\mathrm{A}_{10} \mathrm{M}_{50} \mathrm{~F}_{50} \mathrm{~W}_{\mathrm{m}} \mathrm{I}_{\mathrm{S}}$ due to higher grain yield. The combination of $\mathrm{A}_{10} \mathrm{~F}_{100} \mathrm{~W}_{\mathrm{m}} \mathrm{I}_{\mathrm{S}}$ increased 16.80 per cent higher net income over recommended practice of hybrid rice $\left(\mathrm{A}_{21} \mathrm{~F}_{100} \mathrm{~W}_{\mathrm{H}} \mathrm{I}_{3 \mathrm{D}}\right)$. However, labour productivity was comparable but $\mathrm{B}$ : $\mathrm{C}$ ratio increased in $\mathrm{A}_{10} \mathrm{~F}_{100} \mathrm{~W}_{\mathrm{m}} \mathrm{I}_{\mathrm{S}}$ due to higher economic yield. The labour productivity was highest under $\mathrm{A}_{10} \mathrm{~F}_{100} \mathrm{~W}_{\mathrm{c}} \mathrm{I}_{\mathrm{S}}$ due to the use of chemical which required less number of labours for weeding and also application of chemical fertilizer and irrigation gave higher grain yield. This is accordance with the findings of Thiyagarajan et al. (2002). However, B: C ratio was increased under the treatment of $\mathrm{A}_{10} \mathrm{M}_{50} \mathrm{~F}_{50} \mathrm{~W}_{\mathrm{m}} \mathrm{I}_{\mathrm{S}}$ but at par with $\mathrm{A}_{10} \mathrm{~F}_{100} \mathrm{~W}_{\mathrm{m}} \mathrm{I}_{\mathrm{S}}$. This is due to less input cost and higher economical yield. The lowest net income and BCR was recorded under $\mathrm{A}_{10} \mathrm{M}_{100} \mathrm{~W}_{\mathrm{c}} \mathrm{I}_{\mathrm{S}}$ but at par with $\mathrm{A}_{10} \mathrm{M}_{100} \mathrm{~W}_{\mathrm{m}} \mathrm{I}_{\mathrm{S}}$ and $\mathrm{A}_{14} \mathrm{M}_{100} \mathrm{~W}_{\mathrm{m}} \mathrm{I}_{\mathrm{S}}$.

\section{CONCLUSION}

The combination of 10 days aged seedlings, $100 \%$ nutrients through inorganic sources or $50 \%$ through inorganic $+50 \%$ through organic sources, weeds controlled thrice mechanically by cono weeder and irrigation as per SRI i.e., $2 \mathrm{~cm}$ at hairline crack development stage $\left(\mathrm{A}_{10} \mathrm{~F}_{100} \mathrm{~W}_{\mathrm{m}} \mathrm{I}_{\mathrm{s}}\right.$ or $\left.\mathrm{A}_{10} \mathrm{M}_{50} \mathrm{~F}_{50} \mathrm{~W}_{\mathrm{m}} \mathrm{I}_{\mathrm{s}}\right)$ gave similar and maximum growth characters, yield attributes and yield of hybrid rice. The total water requirement was highest under SRI i.e., 10 days aged seedlings, 100\% nutrients through organics, mechanical weeding and application of $2 \mathrm{~cm}$ water at hairline crack development stage $\left(\mathrm{A}_{10} \mathrm{M}_{100} \mathrm{~W}_{\mathrm{m}} \mathrm{I}_{\mathrm{s}}\right)$ and manipulated SRI $\left(\mathrm{A}_{10} \mathrm{~F}_{100} \mathrm{~W}_{\mathrm{m}} \mathrm{I}_{\mathrm{s}}\right.$ or $\left.\mathrm{A}_{10} \mathrm{M}_{50} \mathrm{~F}_{50} \mathrm{~W}_{\mathrm{m}} \mathrm{I}_{\mathrm{s}}\right)$ than recommended practices of hybrid rice $\left(\mathrm{A}_{21} \mathrm{~F}_{100} \mathrm{~W}_{\mathrm{H}} \mathrm{I}_{3 \mathrm{D}}\right)$. Economic viability of hybrid rice revealed that manipulated SRI $\left(\mathrm{A}_{10} \mathrm{~F}_{100} \mathrm{~W}_{\mathrm{m}} \mathrm{I}_{\mathrm{s}}\right.$ or $\left.\mathrm{A}_{10} \mathrm{M}_{50} \mathrm{~F}_{50} \mathrm{~W}_{\mathrm{m}} \mathrm{I}_{\mathrm{s}}\right)$ proved to be superior over SRI $\left(\mathrm{A}_{10} \mathrm{M}_{100} \mathrm{~W}_{\mathrm{m}} \mathrm{I}_{\mathrm{s}}\right)$ and recommended practices of hybrid rice $\left(\mathrm{A}_{21} \mathrm{~F}_{100} \mathrm{~W}_{\mathrm{H}} \mathrm{I}_{3 \mathrm{D}}\right)$. 


\section{REFERENCES}

Krishna, A. 2000. Effect of age of seedlings on performance of rice (Oryza sativa L.) cultivars under late planted condition. Journal of Research ANGRAU, 28: 73-74

Krishna, A., Biradarpatil, N.K. and Channappagoudar, B.B. 2008. Influence of system of rice intensification (SRI) cultivation on seed yield and quality. Karnataka Journal of Agricultural Sciences, 21(3): 369-372 Lokanadhan, S., Ravichandran, V., Suresh, S., Rabindran, R., Thiyagarajan, K. and Mohanasundaram, K. 2007. Efficient resource utilization in SRI method of rice (CORH 3) cultivation-An analysis. p. 79-81. In Proc. National symposium on SRI in India, $2^{\text {nd }}, 3-5$ October 2007. Tripura, India

Mitra, B.K., Karim, A.J.M.S., Haque, M.M., Ahmed, G.J.U. and Bari, M.N. 2005. Effect of weed management practices on transplanted Aman rice. Journal of Agronomy, 4(3): $238-241$

Padmavati, P., Singh, S. and Prasad, R. 1998. Effect of planting patterns and levels of nitrogen on performance of conventional and hybrid rice variety. p. 109-113 In Proc. First International Agronomy Congress, 23-27 Nov., 1998 at New Delhi, India

Shrirame, M.D., Rajgire, H.J. and Rajgire, A.H. 2000. Effect of spacing and seedling number per hill on growth attributes and yield of rice hybrids under lowland condition. Journal of Soils and Crops, 10(1): 109-113

Thiyagarajan, T.M., Senthikumar, K., Brindavan, P.S., Hengsdijk, H., Ramasumy, S. and Velu, V. 2002. Crop management options for increasing water productivity in rice. Journal of Agricultural Resource Management, 1: 169-181

Uphoff, N. 2001. Understanding SRI? Appropriate Technology, 28(3):12-13

Vijayakumar, M., Ramesh, S., Prabhakaran, N.K., Subbian, P. and Chandrasekaran, B. 2006. Influence of system of rice intensification (SRI) practices on growth characters, days to flowering, growth analysis and labour productivity of rice. Asian Journal of Plant Science, 5(6): 984-989

Wang, S., Cao, W., Jiang, D., Dai, T. and Zhu, Y. 2003. Effect of SRI technique on physiological characteristics and population development in rice. Chinese Journal of Rice Science, 17(1): 31-36 
Table 2: Growth, weed parameters and water requirement of hybrid rice as influenced by system of rice intensification (SRI) and its manipulation (mean data of 2 years)

\begin{tabular}{|c|c|c|c|c|c|c|c|c|c|c|c|}
\hline \multirow[t]{2}{*}{ Treatment } & \multirow{2}{*}{$\begin{array}{l}\text { Plant } \\
\text { height } \\
\text { at } \\
\text { harvest } \\
\text { (cm) }\end{array}$} & \multirow{2}{*}{$\begin{array}{c}\text { No. of } \\
\text { leaves } \\
\text { plant }^{-1} \\
\text { at } 90 \\
\text { DAT }\end{array}$} & \multirow{2}{*}{$\begin{array}{c}\text { No. of } \\
\text { tillers } \\
\text { plant }^{-1} \\
\text { at } \\
\text { harves } \\
\text { t }\end{array}$} & \multirow{2}{*}{$\begin{array}{c}\text { Weigh } \\
t \text { of } \\
\text { tillers } \\
\text { plant }^{-1} \\
\text { at } 90 \\
\text { DAT }\end{array}$} & \multicolumn{2}{|c|}{$\begin{array}{c}\text { Dry weight at } \\
\text { 60 DAT } \\
\left(\text { g plant }^{-1}\right)\end{array}$} & \multirow{2}{*}{$\begin{array}{l}\text { Flag } \\
\text { leaf } \\
\text { Area } \\
\text { at } 65 \\
\text { DAT } \\
\left(\mathrm{cm}^{2}\right)\end{array}$} & \multirow{2}{*}{$\begin{array}{c}\text { Spade } \\
\text { value } \\
\text { of } \\
\text { Flag } \\
\text { leaf at } \\
65 \\
\text { DAT }\end{array}$} & \multirow{2}{*}{$\begin{array}{c}\text { Weed } \\
\text { densit } \\
\text { y at } 54 \\
\text { DAT } \\
(\text { No. } \\
\left.\text { m }^{-2}\right)\end{array}$} & \multirow{2}{*}{$\begin{array}{c}\text { Weed } \\
\text { bioma } \\
\text { ss at } \\
54 \\
\text { DAT } \\
\left(\text { g }_{2}\right)^{-}\end{array}$} & \multirow{2}{*}{$\begin{array}{c}\text { Water } \\
\text { require } \\
\text { ment } \\
(\mathrm{mm})\end{array}$} \\
\hline & & & & & Root & Shoot & & & & & \\
\hline $\mathrm{A}_{10} \mathrm{M}_{100} \mathrm{~W}_{\mathrm{m}} \mathrm{I}_{\mathrm{S}}$ & 98.90 & 106.31 & 32.88 & 4.58 & 10.77 & 35.81 & 42.44 & 37.83 & 27.21 & 13.59 & 823 \\
\hline $\mathrm{A}_{10} \mathrm{M}_{100} \mathrm{~W}_{\mathrm{c}} \mathrm{I}_{\mathrm{S}}$ & 90.67 & 91.82 & 27.75 & 3.34 & 9.21 & 29.86 & 30.17 & 35.33 & 38.34 & 21.66 & 823 \\
\hline $\mathrm{A}_{10} \mathrm{M}_{100} \mathrm{~W}_{\mathrm{m}} \mathrm{I}_{3 \mathrm{D}}$ & 101.67 & 107.25 & 32.10 & 4.69 & 10.89 & 36.22 & 43.10 & 38.16 & 24.33 & 12.87 & 790 \\
\hline $\mathrm{A}_{14} \mathrm{M}_{100} \mathrm{~W}_{\mathrm{m}} \mathrm{I}_{\mathrm{S}}$ & 99.47 & 105.20 & 32.28 & 4.63 & 10.30 & 36.47 & 43.90 & 37.73 & 27.25 & 13.82 & 803 \\
\hline $\mathrm{A}_{14} \mathrm{M}_{100} \mathrm{~W}_{\mathrm{m}} \mathrm{I}_{3 \mathrm{D}}$ & 100.28 & 107.89 & 32.05 & 4.63 & 10.87 & 36.16 & 42.48 & 37.70 & 24.30 & 12.90 & 800 \\
\hline $\mathrm{A}_{10} \mathrm{~F}_{100} \mathrm{~W}_{\mathrm{m}} \mathrm{I}_{\mathrm{S}}$ & 120.10 & 147.78 & 41.28 & 7.30 & 12.61 & 43.56 & 59.09 & 44.80 & 19.79 & 6.18 & 823 \\
\hline $\mathrm{A}_{10} \mathrm{~F}_{100} \mathrm{~W}_{\mathrm{c}} \mathrm{I}_{\mathrm{S}}$ & 108.31 & 131.33 & 37.11 & 6.11 & 11.50 & 39.46 & 52.30 & 41.06 & 24.71 & 10.44 & 823 \\
\hline $\mathrm{A}_{14} \mathrm{~F}_{100} \mathrm{~W}_{\mathrm{m}} \mathrm{I}_{\mathrm{S}}$ & 112.17 & 133.67 & 37.78 & 6.18 & 11.49 & 39.41 & 51.48 & 41.47 & 23.48 & 9.29 & 803 \\
\hline $\mathrm{A}_{10} \mathrm{M}_{50} \mathrm{~F}_{50} \mathrm{~W}_{\mathrm{m}} \mathrm{I}_{\mathrm{S}}$ & 118.73 & 145.20 & 40.91 & 7.29 & 12.60 & 43.44 & 57.91 & 44.55 & 20.78 & 6.29 & 823 \\
\hline $\mathrm{A}_{10} \mathrm{M}_{50} \mathrm{~F}_{50} \mathrm{~W}_{\mathrm{c}} \mathrm{I}_{\mathrm{S}}$ & 101.27 & 107.74 & 33.61 & 4.58 & 10.80 & 35.86 & 42.30 & 37.93 & 27.12 & 13.77 & 823 \\
\hline $\mathrm{A}_{14} \mathrm{M}_{50} \mathrm{~F}_{50} \mathrm{~W}_{\mathrm{m}} \mathrm{I}_{\mathrm{S}}$ & 109.50 & 133.53 & 38.41 & 6.06 & 11.44 & 39.29 & 50.72 & 41.68 & 23.74 & 9.19 & 803 \\
\hline $\mathrm{A}_{21} \mathrm{~F}_{100} \mathrm{~W}_{\mathrm{H}} \mathrm{I}_{3 \mathrm{D}}$ & 108.87 & 66.33 & 18.65 & 3.28 & 5.94 & 20.17 & 50.25 & 40.35 & 26.28 & 13.97 & 800 \\
\hline $\mathrm{S} \mathrm{Em} \pm$ & 2.14 & 1.52 & 0.91 & 0.52 & 0.53 & 1.23 & 1.17 & 0.91 & 0.86 & 1.11 & - \\
\hline $\mathrm{CD}(\mathrm{P}=\mathbf{0 . 0 5})$ & 4.42 & 3.14 & 1.89 & 1.08 & 1.09 & 2.53 & 2.41 & 1.89 & 1.78 & 2.31 & - \\
\hline
\end{tabular}

A-Age of seedling; M-Manure; F-Fertilizer; $\mathrm{W}_{\mathrm{m}}, \mathrm{W}_{\mathrm{c}} \& \mathrm{~W}_{\mathrm{H}^{-}}$- Mechanical, chemical \& hand weeding, respectively; $\mathrm{I}_{\mathrm{S}} \& \mathrm{I}_{3 \mathrm{D}^{-}}$Irrigation as per SRI \& at $3 \mathrm{DAD}$ 
Table 3: Yield attributes, yield and economics of hybrid rice as influenced by system of rice intensification (SRI) and its manipulation ( pooled data of 2 years)

\begin{tabular}{|c|c|c|c|c|c|c|c|c|c|c|}
\hline Treatment & $\begin{array}{l}\text { Effective } \\
\text { panicles } \\
\left(\mathrm{No.m}^{-2}\right)\end{array}$ & $\left.\begin{array}{c}\text { Weight } \\
\text { of } \\
\text { panicles }\end{array}\right)\left(\begin{array}{l}\text { g } \\
\left.\text { panicle }^{-1}\right)\end{array}\right.$ & $\begin{array}{c}\text { Fertile } \\
\text { grains } \\
\text { (No.) }\end{array}$ & $\begin{array}{c}\text { Sterility } \\
(\%)\end{array}$ & $\begin{array}{l}\text { Weight } \\
\text { of } 1000- \\
\text { seed }(\mathrm{g})\end{array}$ & $\begin{array}{c}\text { Grain } \\
\text { yield } \\
\left(\mathrm{t} \text { ha }^{-1}\right)\end{array}$ & $\begin{array}{c}\text { Total cost } \\
(000 \text { 'Rs. } \\
\left.\text { ha }^{-1}\right)\end{array}$ & $\begin{array}{c}\text { Gross } \\
\text { margin } \\
\left(000^{\prime} R s .\right. \\
\left.\text { ha }^{-1}\right)\end{array}$ & BCR & $\begin{array}{c}\text { Labour } \\
\text { productivity } \\
\text { Rs. Output } \\
\text { Rs.input }^{-1} \text { ) }\end{array}$ \\
\hline $\mathrm{A}_{10} \mathrm{M}_{100} \mathrm{~W}_{\mathrm{m}} \mathrm{I}_{\mathrm{S}}$ & 292.48 & 4.18 & 141.18 & 29.99 & 22.57 & 5.99 & 21.40 & 35.01 & 1.64 & 5.82 \\
\hline $\mathrm{A}_{10} \mathrm{M}_{100} \mathrm{~W}_{\mathrm{c}} \mathrm{I}_{\mathrm{S}}$ & 253.12 & 3.22 & 125.50 & 33.15 & 22.09 & 5.20 & 19.30 & 30.15 & 1.56 & 8.16 \\
\hline $\mathrm{A}_{10} \mathrm{M}_{100} \mathrm{~W}_{\mathrm{m}} \mathrm{I}_{3 \mathrm{D}}$ & 289.80 & 4.14 & 139.83 & 30.18 & 22.58 & 5.98 & 20.72 & 35.61 & 1.72 & 5.81 \\
\hline $\mathrm{A}_{14} \mathrm{M}_{100} \mathrm{~W}_{\mathrm{m}} \mathrm{I}_{\mathrm{S}}$ & 290.35 & 4.06 & 136.89 & 30.08 & 22.56 & 5.94 & 21.40 & 34.54 & 1.61 & 5.77 \\
\hline $\mathrm{A}_{14} \mathrm{M}_{100} \mathrm{~W}_{\mathrm{m}} \mathrm{I}_{3 \mathrm{D}}$ & 287.64 & 4.10 & 138.01 & 30.05 & 22.55 & 5.95 & 20.72 & 35.31 & 1.70 & 5.74 \\
\hline $\mathrm{A}_{10} \mathrm{~F}_{100} \mathrm{~W}_{\mathrm{m}} \mathrm{I}_{\mathrm{S}}$ & 342.72 & 5.94 & 175.33 & 25.02 & 23.48 & 7.52 & 22.62 & 47.21 & 2.09 & 7.20 \\
\hline $\mathrm{A}_{10} \mathrm{~F}_{100} \mathrm{~W}_{\mathrm{c}} \mathrm{I}_{\mathrm{S}}$ & 303.84 & 4.76 & 156.11 & 29.33 & 23.07 & 6.54 & 20.47 & 40.92 & 2.00 & 10.14 \\
\hline $\mathrm{A}_{14} \mathrm{~F}_{100} \mathrm{~W}_{\mathrm{m}} \mathrm{I}_{\mathrm{S}}$ & 309.92 & 4.85 & 158.56 & 28.12 & 23.17 & 6.83 & 22.62 & 41.10 & 1.82 & 6.57 \\
\hline $\mathrm{A}_{10} \mathrm{M}_{50} \mathrm{~F}_{50} \mathrm{~W}_{\mathrm{m}} \mathrm{I}_{\mathrm{S}}$ & 324.80 & 5.82 & 172.13 & 25.24 & 23.43 & 7.39 & 21.99 & 46.82 & 2.13 & 7.10 \\
\hline $\mathrm{A}_{10} \mathrm{M}_{50} \mathrm{~F}_{50} \mathrm{~W}_{\mathrm{c}} \mathrm{I}_{\mathrm{S}}$ & 293.48 & 4.16 & 145.78 & 30.13 & 22.56 & 6.01 & 19.88 & 36.64 & 1.84 & 9.33 \\
\hline $\mathrm{A}_{14} \mathrm{M}_{50} \mathrm{~F}_{50} \mathrm{~W}_{\mathrm{m}} \mathrm{I}_{\mathrm{S}}$ & 311.52 & 4.80 & 156.51 & 28.04 & 23.15 & 6.81 & 21.99 & 41.60 & 1.89 & 6.56 \\
\hline $\mathrm{A}_{21} \mathrm{~F}_{100} \mathrm{~W}_{\mathrm{H}} \mathrm{I}_{3 \mathrm{D}}$ & 304.85 & 4.71 & 156.50 & 30.03 & 23.05 & 6.50 & 21.76 & 39.28 & 1.81 & 7.13 \\
\hline $\mathrm{S} E \mathrm{Em} \pm$ & 4.16 & 0.14 & 4.49 & 0.85 & 0.18 & 0.16 & - & 1.40 & 0.04 & 0.16 \\
\hline CD $(P=0.05)$ & 8.60 & 0.29 & 9.29 & 1.76 & 0.37 & 0.33 & - & 2.90 & 0.10 & 0.33 \\
\hline
\end{tabular}

A-Age of seedling; M-Manure; F-Fertilizer; $\mathrm{W}_{\mathrm{m}}, \mathrm{W}_{\mathrm{c}} \& \mathrm{~W}_{\mathrm{H}}-$ Mechanical, chemical \& hand weeding, respectively; $\mathrm{I}_{\mathrm{s}} \& \mathrm{I}_{3 \mathrm{D}^{-}}$Irrigation as per $\mathrm{SRI} \&$ at $3 \mathrm{DAD}$ 